Francisco J. Navarro-Brull*, Pedro Poveda, Rubén Ruiz-Femenia, Pedro Bonete, Jaime Ramis and Roberto Gómez*

\title{
Guidelines for the design of efficient sono-microreactors
}

\begin{abstract}
Possible drawbacks of microreactors are inefficient reactant mixing and the clogging of microchannels when solid-forming reactions are carried out or solid (catalysts) suspensions are used. Ultrasonic irradiation has been successfully implemented for solving these problems in microreactor configurations ranging from capillaries immersed in ultrasonic baths to devices with miniaturized piezoelectric transducers. Moving forward in process intensification and sustainable development, the acoustic energy implementation requires a strategy to optimize the microreactor from an ultrasound viewpoint during its design. In this work, we present a simple analytical model that can be used as a guide to achieving a proper acoustic design of stacked microreactors. An example of this methodology was demonstrated through finite element analysis and it was compared with an experimental study found in the literature.
\end{abstract}

Keywords: acoustic modeling; microreactor design; optimization; process intensification; sonochemistry.

DOI 10.1515/gps-2014-0052

Received July 15, 2014; accepted August 22, 2014

\section{Introduction}

Microreactors, also known as Lab on a Chip, can exhibit significant advantages over classical reactors because the reaction conditions in the microchannels noticeably differ from those in large-scale reactors. By reducing the diameter of the reactor channels to tens/hundreds of micrometers,

*Corresponding authors: Francisco J. Navarro-Brull and Roberto Gómez, Institut Universitari d'Electroquímica i Departament de Química Física, Apartat 99, E-03080 Alicante, Spain, Tel.: +34965903748, e-mail: fj.navarro@ua.es, roberto.gomez@ua.es. http://orcid.org/0000-0002-5231-8032 (Roberto Gómez)

Pedro Poveda and Jaime Ramis: Departament de Física, Enginyeria de Sistemes i Teoria del Senyal, Alicante, Spain Rubén Ruiz-Femenia: Departament d’Enginyeria Química, Universitat d'Alacant, Alicante, Spain

Pedro Bonete: Institut Universitari d'Electroquímica i Departament de Química Física the flow conditions change radically. On this scale, laminar flow (i.e., low Reynolds number) is obtained and phenomena such as diffusion, among others, become decisive. In addition, the high increase of the surface-to-volume ratio allows them to sustain fast exothermic reactions under controlled conditions, which can significantly improve organic synthetic processes or other applications [1]. Thus, microfluidic technology leads to green and economical production methods due to a higher selectivity of target products and a reduced amount of waste. It is thus not surprising that microreactors have been facing an increasing demand in the fine chemistry sector and, more specifically, in the pharmaceutical and food industry. New lines of research are focused on feasible solutions for their scale-up (usually by numbering-up) as well as on the use of innovative (an efficient) energy sources such as microwave, ultrasound, and plasma [2].

Yet, the handling of solids (catalysts, reagents, products, and by-products) is one of the main drawbacks in the chemical reactions carried out in microreactors. During a reaction, the microchannels can be irreversibly clogged by the formation of solid aggregates or precipitates. In addition, microreactors can suffer from inefficient reactant mixing due to the predominance of laminar flow.

The use of ultrasonic irradiation has been successfully implemented not only to prevent these problems because of its well-known mixing effect, but also because of mass transport enhancement or solid catalyst reactivation [39]. Several configurations ranging from immersion of the capillaries in ultrasonic baths [10] to the integration of miniaturized piezoelectric transducers in the microchannel plate have been used for this purpose [11]. It is worth noting that the acoustic field generated in the microreactor strongly depends on its configuration [12] (i.e., the construction materials, sizes and geometries of the different parts, etc.). However, the complexity that ultrasonic irradiation and its optimization involve is usually not regarded as being determinant, because its effects are a tool, not an end. In this respect, there are only a few examples in the literature where the influence of the position of the piezoelectric material, and the sizing and geometry of the microreactor have been considered [13, 14]. 
It must be noted that ultrasound irradiation (i.e., sonication) involves several physicochemical effects, many of them related to acoustic cavitation [15], which strongly depend on the working frequency of the transducer [16]. Consequently, an understanding of all these variables and their influence should be taken into account when optimizing the effects of ultrasound instead of just adjusting the applied frequency once the device is mounted [6].

This work presents a simplified strategy (Figure 1) to optimize a stack type microreactor from an ultrasonic viewpoint (intense and homogeneous acoustic field) in the desired frequency range. First, a classic and wellknown 1D equation will provide information about the proper sizing of the device as a function of the working frequency (including the separation between the transducer and the desired sonication area). Second, the problem will be addressed by using a finite element method (FEM) in 2D simulations for modeling the acoustic field inside the reactor, the vibrations of the solid and the electromechanical properties of the transducer.

In order to substantiate the proposed strategy and its simulation results, we have chosen from the literature as an example a Teflon homemade stack microreactor with an integrated piezoelectric actuator used to prevent the clogging of microchannels (see Figure 2).

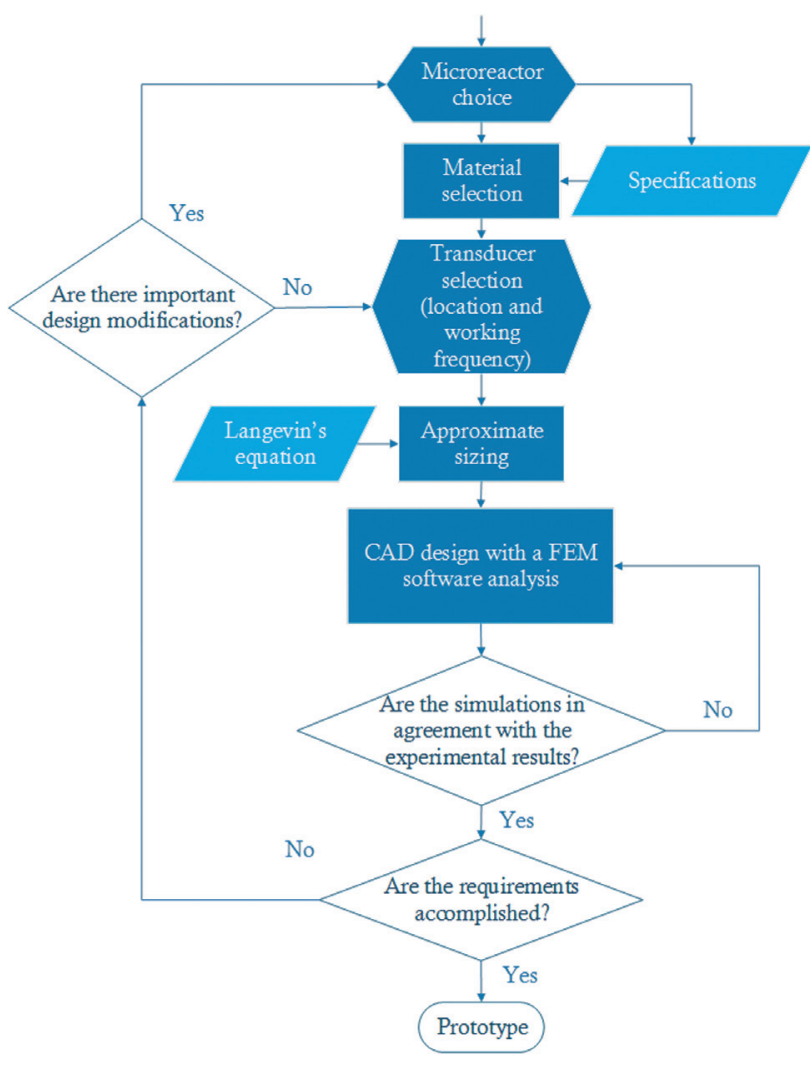

Figure 1 Guideline proposed for the design of sono-microreactors.
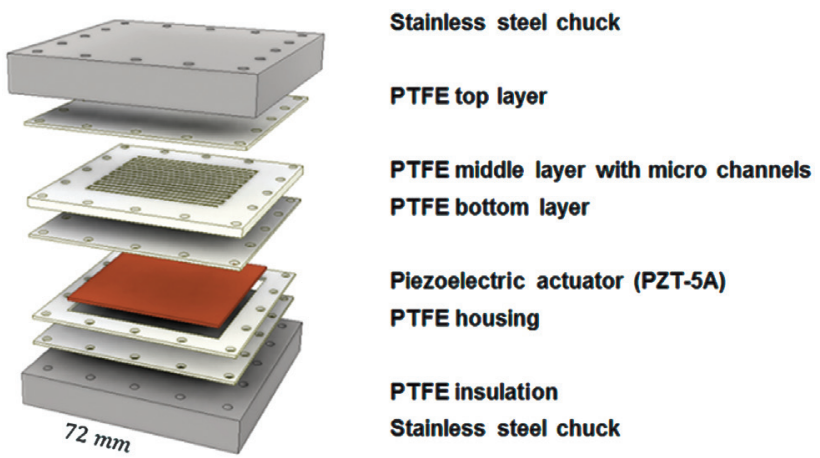

Figure 2 Representation of the assembly constituting the Teflonstacked microreactor proposed in the literature.

Reproduced with permission from Kuhn et al. [6]. Copyright 2011 The Royal Society of Chemistry.

\section{Review of the experimental results}

A Teflon stack microreactor (Figure 2) with an integrated piezoelectric actuator was proposed in [6] to study the advantage of ultrasound irradiation, i.e., microchannel clogging prevention. The device was fabricated by assembling polytetrafluoroethylene (PTFE) plates of $70 \times 70 \mathrm{~mm}$, one of them with engraved microchannels with $600 \mu \mathrm{m}$ in width, and by compressing them between two stainless steel chucks. A piezoelectric sheet of $1 \mathrm{~mm}$ thickness was encased in one of the PTFE plates and was driven at different frequencies by a wave generator and an amplifier at a load power of $30 \mathrm{~W}$. The resulting sono-microreactor was tested under microflow conditions to carry out Pd-catalyzed couplings of anilines and aryl halides. Inorganic byproducts, such as $\mathrm{NaCl}$ and $\mathrm{NaBr}$, precipitate instantly due to their insolubility in the non-polar solvent where these reactions take place. Hence, the microchannels where the reaction occurs are prone to be clogged by these solids, which makes ultrasound irradiation a necessity.

Kuhn et al. [6] first documented the formation of cavitation bubbles by means of a spiral microreactor with an actuator that was glued on its backside. With this device, an optimum frequency of $50 \mathrm{kHz}$ was found - in terms of particle size distribution - within a wide range of frequencies $(20 \mathrm{kHz}-3 \mathrm{MHz})$. Afterward, experiments were performed with the Teflon stack microreactor (Figure 2), again with a focus on the particle size distribution. The median particle diameter was 20,8 and $12 \mu \mathrm{m}$ upon sonication at 40, 50, and $60 \mathrm{kHz}$, respectively. Within this narrower range of frequencies, an optimum ultrasound frequency of $50 \mathrm{kHz}$ was a new found for the Teflon stack microreactor [6]. In any case, we believe that the stacked 
microreactor under consideration should be considered as an important proof-of-concept rather than as a fully optimized sono-microreactor. In the next sections, we will provide guidelines for addressing the acoustic optimization of such devices.

\section{Acoustic sizing optimization}

The placement of the transducer within the microreactor is not trivial. If it is placed without adequate consideration, the resonance frequency of the system can be far from the natural oscillation frequency of the piezoelectric material and could even produce some damages in its structure. Besides, the maximum acoustic pressure can be localized at a suboptimal point, far from the microchannel(s), i.e., the area of interest. In the end, an improper placement of the transducer would cause a poor conversion of the electric power into acoustic pressure. All these drawbacks can be roughly dealt with by using the Langevin's model, which allows for the sizing of the different stacks of the microreactor once the materials of the sono-microreactor and the desired working frequency have been chosen.

\subsection{Langevin's transducer}

Prestressed sandwich transducers, or Langevin's transducers [17-19], are frequently applied in the high-power ultrasound manufacturing industry as narrow-band piezoelectric transducers. Their basic structure consists of one or more pairs of piezoceramic rings, enclosed by metal endings and mechanically compressed by a central bolt. This half-wave resonant structure vibrates in its longitudinal or axial direction. A representation of the most common Langevin's kind of transducer - often called a symmetrical transducer - is shown in Figure 3.

Every Langevin's transducer can be divided into its three inherent parts, namely:

- the reflector, representing the back side of the transducer and looking for the maximum reflection of the acoustic wave;

- the actuator, usually made of a lead-zirconatetitanate ceramic (PZT);

- the emitter, transfering oscillations to the operating medium.

The reflector and emitter are represented in Figure 3 with the letters $r$ and $e$, respectively.

Sandwich transducer design and optimization do necessarily involve different materials and knowledge of

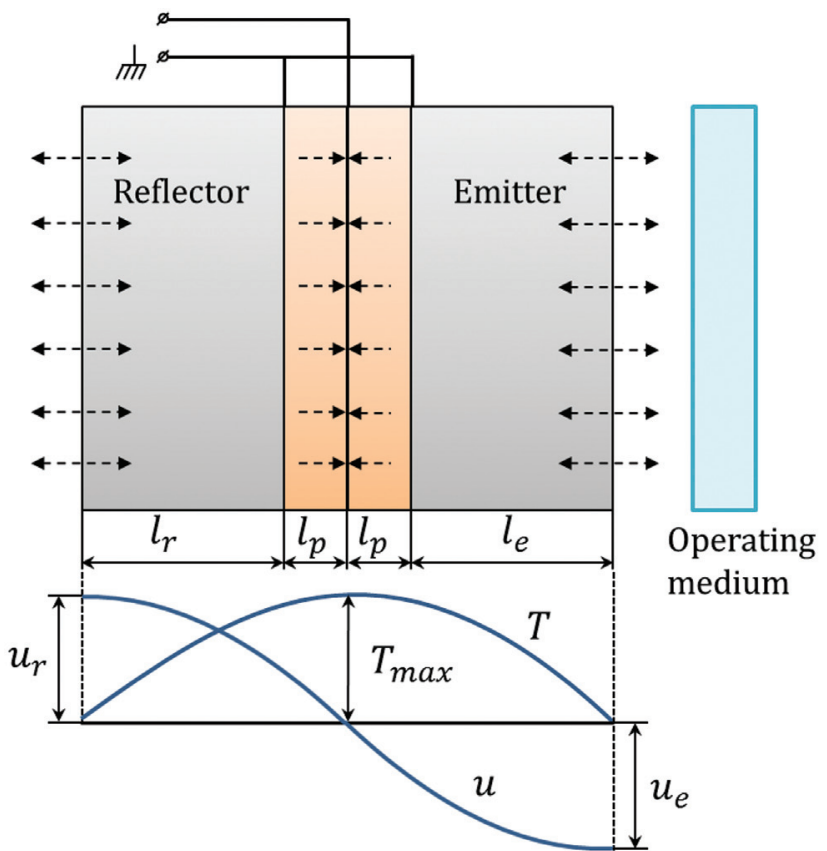

Figure 3 Half-wave transducer with same material endings and cross-section areas showing its displacement amplitude $(u)$ and mechanical stress $(T)$ curves [20].

mechanics, acoustics, and electronics. The next section will highlight one of the simplest one-dimensional models achieving approximate results despite its simplicity.

\subsection{Langevin's equation}

Although several one-dimensional approaches can be found in the literature, Langevin's equation constitutes the most frequently applied equation for the design of sandwich transducers. Langevin's equation is widely used due to its simplicity and flexibility. However, it should be only applied provided that the transducer has the same cross-sections at both ends (usually called symmetric transducers) [21, 22]. In any case, what all these one-dimensional models have in common is that they enable the possibility of determining the transducer dimensions for a specific resonant frequency by using an equivalent circuit analogy. In fact, a mechanical system can be described by its electrical analog, and viceversa. KLM and Mason's models are used to design the transducer by simplifying the wave equation solution in the model. This is possible thanks to an equivalent electrical network representation used for calculating electromechanical parameters [17, 23, 24]. Thus, a Langevin-type transducer can be modeled under the following assumptions: 
- There are solely plane-wave propagations along the longitudinal section of the reactor, where the tension in the interior of the pieces is evenly distributed throughout its cross section.

- The forces exerted by the back and front ends on the air are considered as negligible and the equivalent circuit is simplified to a single impedance.

When the system vibrates at the resonance frequency, the impedance of such an equivalent circuit becomes zero, leading to Equation 1 for a half-wave resonant structure of a Langevin-type ultrasonic transducer:

$$
k_{p} l_{p}+\tan ^{-1}\left(\frac{Z_{r}^{c}}{Z_{p}^{c}} \tan k_{r} l_{r}\right)+\tan ^{-1}\left(\frac{Z_{e}^{c}}{Z_{p}^{c}} \tan k_{f} l_{e}\right)=\pi
$$

where $k$ denotes the angular wavenumber $(2 \pi / \lambda$, with $\lambda$ being the wavelength) and $Z$ the acoustic impedance ( $\rho c$, with $\rho$ being the material density and $c$ the sound velocity) of each section.

The first term of Equation 1 corresponds to the piezoelectric crystal, and the second and third terms to the back and front side, respectively. Consequently, $l_{p}$ is the thickness of the piezoelectric material, while $l_{r}$ is the thickness of the Teflon layer behind the piezo (reflector) and $l_{e}$ is that of the layer before it (emitter). It is necessary to define the size of the piezoelectric ceramic because it should be located at a vibration node [25] allowing the division of the transducer into two sections, where each one will develop a quarter of the wavelength of the resonator (Figure 3). In this way, Equation 1 can be splitted into Equations 2 and 3 (Figure 3).

$$
k_{p} l_{p}+\tan ^{-1}\left(\frac{Z_{r}^{c}}{Z_{p}^{c}} \tan k_{r} l_{r}\right)=\frac{\pi}{2}
$$

$$
\tan ^{-1}\left(\frac{Z_{e}^{c}}{Z_{p}^{c}} \tan k_{f} l_{e}\right)=\frac{\pi}{2}
$$

Finally, it must be underlined that the two stainless steel chucks closing the microreactor have not been considered in this sizing. The transducer is modeled to have a maximum output at the back side where, due to the impedance differences between Teflon and stainless steel, the transmission of the vibration will be insignificant ( $97 \%$ of reflection). In contrast, on the front side, the width of the Teflon is designed to have a pressure antinode (maximum acoustic pressure) at the microchannel. The interface between Teflon and stainless steel on the front side will be located at a node, where the vibration is zero and therefore not interfering with the signal. Figure 4 shows sizing results from the Langevin model (Equations 2 and 3) applied to the device described in Figure 2 at different working frequencies.

Let us consider a sono-microreactor that should operate at $50 \mathrm{kHz}$, which is the optimum frequency in the stacked sono-microreactor studied by Kuhn et al. [6]. As in the latter case, PTFE will be considered as a construction material. The thicknesses of the PTFE layers in contact with the piezoelectric $\left(l_{r}\right.$ and $\left.l_{e}\right)$, calculated with Equations 2 and 3 for $50 \mathrm{kHz}$, are 2.12 and $2.13 \mathrm{~mm}$, respectively. These values differ from the original dimensions employed in the literature microreactor, which were 1 and $3.9 \mathrm{~mm}$, respectively. The difference between the original microreactor [6] and its corresponding acoustic design following Langevin's equation is illustrated in Figure 5. Despite the dimensions being alike, the difference between both of them is not surprising. In this case, we are adopting a traditional 1D longitudinal equation as a starting point in the design. Among the many approximations carried out, we highlight the
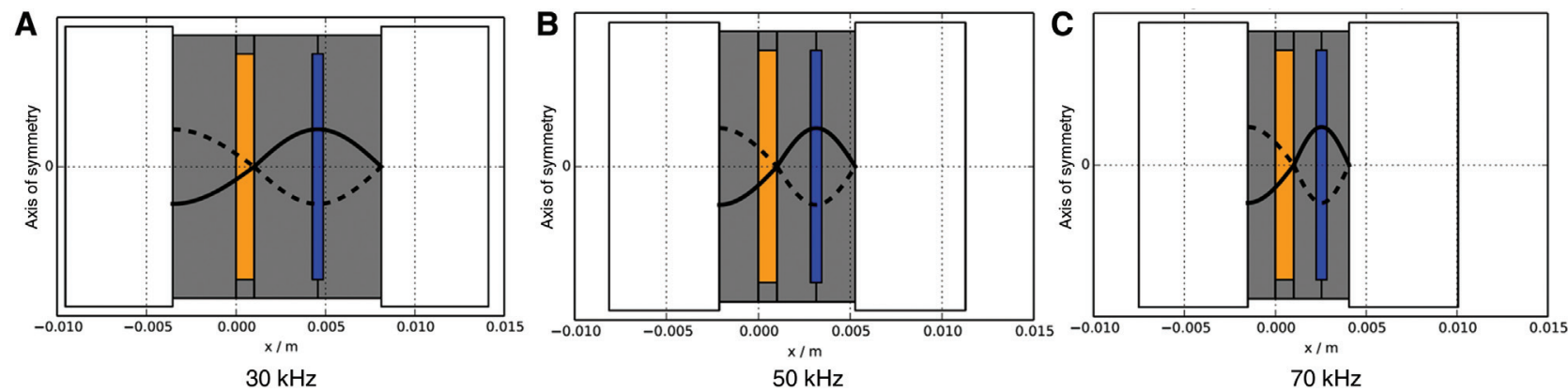

Figure 4 Sizing according to the Langevin's equation for different working frequencies applied to a Teflon-stacked microreactor where the antinode (maximum acoustic pressure) is located at the desired place (reaction liquid).

White blocks refer to stainless steel, gray to PTFE, blue to the reactant liquid and orange to PZT-5A. Solid and dashed black lines represent the standing waves being formed along the Teflon microreactor. For the animated version of this figure, please see http://bit.ly/interactive-langevin. 


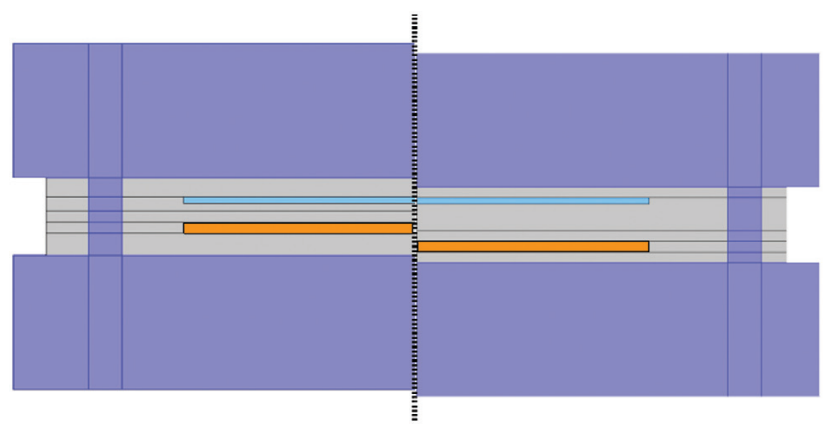

Figure 5 Sketch comparing the dimensions of the Langevin's equation (left) and the original microreactor designs (right).

Dark blue refers to stainless steel, gray to PTFE, blue to the reactant liquid, and orange to PZT-5A.

absence of vibration along the rest of the spatial dimensions. This consideration is valid if the radial dimension is significantly inferior to the longitudinal one, which is not the case. In addition, the experimental study was limited to only three frequencies, among which the qualitative effect of ultrasound irradiation was compared. This together with the limited precision in the design of the literature microreactor helps explain why the dimensions are relatively different, even when the optimum frequency is the same.

Admittedly, analytical models using electromechanical equivalent circuits can be a good starting point, but they do show some limitations [22]. The optimization of sono-microreactors necessarily requires more advanced numerical methods in order to obtain a proper understanding of the complexity that these sonochemical systems involve [26]. The next section will establish a framework leading to a more precise simulation of the acoustic pressure, vibrational modes as well as the strain and stress distributions.

\section{Numerical model (2D)}

The acoustic field within the liquid microchannel and the deformation of the stacked microreactor have been calculated by following simulation strategies found in the literature $[27,28]$.

\subsection{Linear acoustics in the working liquid}

The governing equations for a compressible lossless fluid medium are the momentum equation and the continuity equation. Assuming mono-harmonic vibrations, the wave equation for acoustic waves can be reduced to Equation 4, known as the Helmholtz equation:

$$
\nabla^{2} p+\left(\frac{\omega}{c}\right)^{2} p=0
$$

where $p$ is the acoustic pressure, $c$ is the speed of sound in the liquid and $\omega$ is the angular frequency. At the channel wall, the continuity condition has been applied as a boundary condition to set the interaction from fluid to solid and vice versa (Equation 5).

$$
\frac{1}{\rho_{l} \omega^{2}} \nabla p \cdot \mathbf{n}=\mathbf{u} \cdot \mathbf{n}
$$

where $\mathbf{n}$ is the outward-pointing unit normal vector seen from the inside of the solid domain, $\rho_{l}$ is the density of the liquid, and $\mathbf{u}$ is the structural complex amplitude of the displacement field of the solid. Hence, at the boundaries where the fluid interacts with the solid, there is continuity of the normal component of acceleration: the normal component of the acceleration associated with the acoustic pressure on the boundary is equal to the normal component of the acceleration based on the second derivatives of the structural displacements (mono-harmonic vibrations are assumed for the solid).

\subsection{Vibration of the solid}

Considering that the linear elastic materials present in the microreactor (PTFE and stainless steel) have the same properties in all directions (isotropic), the vibrations transmitted through these solids are calculated by Equation 6 neglecting volume forces and assuming elastic deformations for all materials as well as mono-harmonic vibrations:

$$
-\rho_{s} \omega^{2} \mathbf{u}=\nabla \cdot \boldsymbol{\sigma}
$$

where $\rho_{s}$ is the density of the solid and $\boldsymbol{\sigma}$ is the elastic stress tensor given by Equation 7:

$$
\boldsymbol{\sigma}=\frac{E v}{(1+v)(1-2 v)}(\operatorname{Tr} \varepsilon) \boldsymbol{I}+\frac{E}{1+v} \varepsilon
$$

where $E$ refers to the Young's modulus, $v$ is the Poisson's ratio, $\boldsymbol{I}$ the identity Tensor and $\mathrm{Tr}$ the trace operator. The total strain tensor, $\boldsymbol{\varepsilon}$, can be written in terms of the displacement gradient (Equation 8):

$$
\varepsilon=\frac{1}{2}\left(\nabla \mathbf{u}+\nabla \mathbf{u}^{\top}\right)
$$

As boundary conditions for the solid, the continuity at solid-solid boundaries has been applied, whereas at the 
boundaries in contact with air, a free condition is assumed, - i.e., components of stress equal to zero. At liquid/solid interfaces, the stress imposed on the solid is equal to the normal pressure stress exerted by the liquid (Equation 9).

$$
-\boldsymbol{\sigma} \cdot \mathbf{n}=p_{t} \mathbf{n}
$$

where $p_{t}=p+p_{0}$ is the total pressure evaluated at the interface. Finally, a fixed constraint $(\mathbf{u}=\mathbf{0})$ is set at the ends of the bolts. A loss (damping) factor was added to this model to reproduce the inherent damping of the different materials. In particular, the isotropic loss factor used is the ratio of the loss modulus and the Young's storage modulus. In the frequency domain, the stress relaxation function of a viscoelastic material is represented by $\left(1+j \eta_{s}\right) \mathbf{C}, \mathbf{C}$ being the right Cauchy-Green tensor, $\eta_{s}$ the isotropic loss factor and $j$ the imaginary unit.

\subsection{Piezoelectric material}

The piezoelectric is the actuator of the system, transforming an electric voltage into mechanical energy. Within a piezoelectric, a coupling exists between stress, strain, electric field, and electric displacement, which can be expressed in the stress-charge form by Equations 10 and 11:

$$
\begin{aligned}
& \sigma=c_{E} \varepsilon-e^{\top} \mathbf{E} \\
& \mathbf{D}=e \varepsilon+\epsilon_{s} \mathbf{E}
\end{aligned}
$$

where, $c_{E}$ is the elasticity matrix, $e$ is the coupling matrix, $\mathbf{E}=-\nabla V$ is the electric field, $\mathbf{D}$ is the electric displacement field, and $\epsilon_{s}$ is the permittivity matrix. To make the variables consistent with previous equations, the typical nomenclature has been changed, i.e., the strain is named $\varepsilon$ instead of $S$, and the stress is named $\sigma$ instead of $T$. The ground $(\mathrm{V}=0)$ is placed on the front of the piezoelectric, whereas at the back, an electric potential of $40 \mathrm{~V}$ is applied.

\section{Results and discussion}

Mirroring the results shown in the literature [6], a working frequency of $50 \mathrm{kHz}$ was chosen as the initial design parameter. By respecting the materials and settings of the original design, Equations 2 and 3 provided information about the proper sizing of the device as a function of the working frequency. The dimensions obtained following this procedure where described at the end of Section 3 and compared with other working frequencies (Figure 4). However, these dimensions obtained with the Langevin's equation are only approximations because of the limitations of this model. Simulations according to the model defined in Section 4 represent a step forward for the design of sono-microreactors.

Once the sizing is done according to the Langevin's equation, the model can be numerically solved with a commercial FEM software named COMSOL Multiphysics $^{\mathrm{TM}}$ (version 4.3a - COMSOL AB, Stockholm, Sweden), where the Acoustic-Piezoelectric Interaction Interface was employed in order to obtain the spatial distribution of the acoustic pressure in the working fluid and the deformation of the microreactor.

A frequency scan was carried out between $30 \mathrm{kHz}$ and $70 \mathrm{kHz}$ with steps of $100 \mathrm{~Hz}$ to test the effectiveness of the optimum sizing described by Langevin's equation. The piezoelectric material was PZT-5A. The properties of the liquid in the microreactor tube as well as those of Teflon and stainless steel are given in Table 1. A 2D structured mesh with about 20.000 2nd-order Lagrange elements was used for spatial discretization of a cross-sectional plane. To ensure a proper resolution of the steep gradients occurring in the system displacements, the mesh was refined in the vicinity of the PZT and the reactant liquid. Thus, the maximum element size in these domains was set to $0.3 \mathrm{~mm}$, whereas it did not exceed $1 \mathrm{~mm}$ of size in the rest of the domains. In any case, the number of degrees of freedom (DOFs) per wavelength was in all cases larger than 5 , which ensures a good accuracy.

Figure 6 shows a maximum of acoustic pressure close to $50 \mathrm{kHz}$ (actually at $53.5 \mathrm{kHz}$ ) - i.e., the frequency chosen to perform the acoustic sizing - by simply using the Langevin's equation. However, the evaluation of the acoustic pressure gained in the liquid domain allows seeing that different vibration modes appear at different working frequencies. Concretely, in this spectrum we can distinguish among others two additional major peaks at 41.7 and $59.0 \mathrm{kHz}$, with relatively high maximum and average pressures. Besides, we observe how a frequency shift of a few $\mathrm{kHz}$ can modify significantly the acoustic pressure to be obtained. This kind of information may become important

Table 1 Material properties considered for simulations.

\begin{tabular}{llrl}
\hline Symbol & Description & Value & Units \\
\hline$\rho_{l}$ & Density of liquid & 1000 & $\mathrm{~kg} \cdot \mathrm{m}^{-3}$ \\
$c$ & Sound velocity (liquid) & 1500 & $\mathrm{~m} \cdot \mathrm{s}^{-1}$ \\
$E_{\text {PTFE }}$ & Young's modulus (Teflon) & 0.49 & $\mathrm{GPa}$ \\
$v_{\text {PTFE }}$ & Poisson's ratio (Teflon) & 0.46 & - \\
$\eta_{\text {PTFE }}$ & Isotropic loss factor (Teflon) & 0.042 & - \\
$E_{\text {St. Steel }}$ & Young's modulus (St. Steel) & 205 & $\mathrm{GPa}$ \\
$v_{\text {St. Steel }}$ & Poisson's ratio (St. Steel) & 0.27 & - \\
$\eta_{\text {St. Steel }}$ & Isotropic loss factor (St. Steel) & 0.016 & - \\
\hline
\end{tabular}




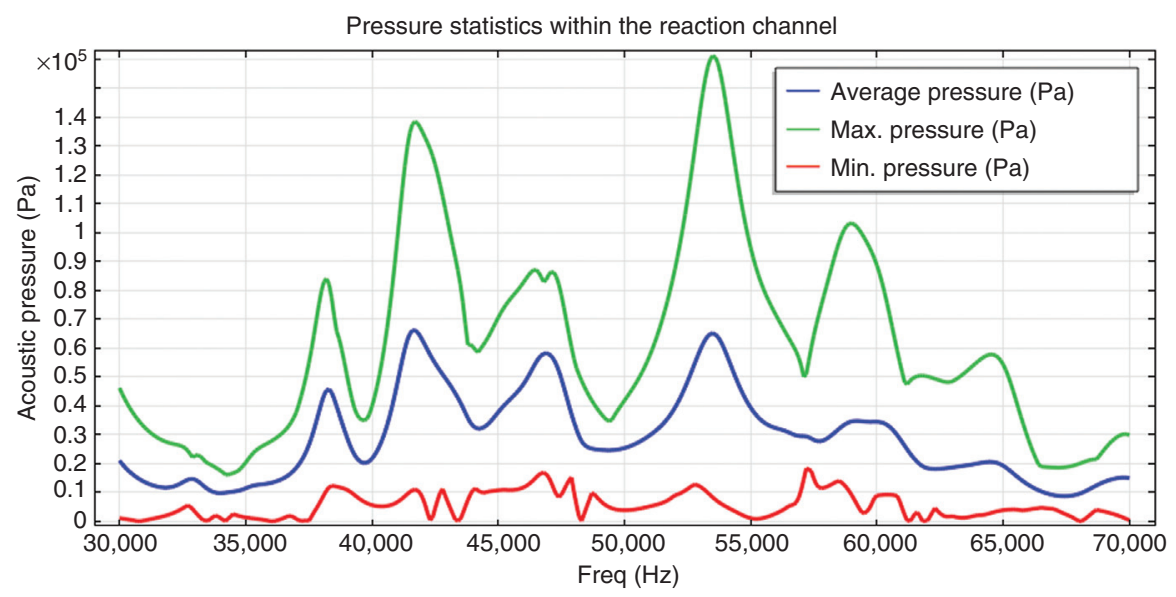

Figure 6 Numerical acoustic pressure vs. frequency numerical results obtained in the reactant liquid, showing a maximum at $53.5 \mathrm{kHz}$. The sizing according to Langevin's equation (analytical model) corresponds to $50 \mathrm{kHz}$.

since the behavior of the transducer may vary with time. For instance, mechanical energy losses will lead to an increase in temperature. A heat evacuation system and a proper control of the working frequency may reduce these non-linear phenomena not considered in the model. In this respect, another interesting option is to try to generate the ultrasound waves as intermittent pulses, a.k.a. pulsed ultrasound.

On the other hand, the impact of the geometry and its complexity can also be analyzed. For example, a completely different spectrum was generated by doing this frequency scan study with a model respecting the original sizing found in the literature [6]. Even when performing this study in a 3D-model - i.e., incorporating additional vibration modes that are overlooked in a $2 \mathrm{D}$-model - the spectrum obtained shows a main peak nearby $40 \mathrm{kHz}$ rather than at $50 \mathrm{kHz}$ as expected from the experimental results. However, it should not be disregarded that the microreactor found in the literature was home-built and presented unavoidable irregularities, e.g., non-ideal material couplings and discontinuities. Consequently, a perfect agreement of the simulation results with the data found in the literature is rather unlikely.

Up to this point, we have only analyzed statistical information - i.e., maxima and averages - of the acoustic pressure in the microchannel. There is however a plethora of information provided by the FEM simulations. Three examples of the spatial distribution of the acoustic pressure and solid deformations are shown in Figure 7. Such responses correspond to the main global resonances of the frequency scan for the model system (sized according to the 1D Langevin's equation at $50 \mathrm{kHz}$ ), which presents a main peak at $53.5 \mathrm{kHz}$ (Figure 7B), and two secondary ones at 41.7 and $59.0 \mathrm{kHz}$ (Figures 7A and C, respectively). In order to facilitate the visualization, color scales were maintained equal. A quick comparison reveals two different behaviors regarding the deformation of the solids (gray scale). The response of the system at $53.5 \mathrm{kHz}$ (Figure 7B) shows mainly longitudinal deformations, which is in contrast with the other two cases. This is in agreement with the fact that this frequency is the closest to that assumed when sizing the device by means of the Langevin's model. However, the pressure field observed in the liquid (colored scale) is far from homogeneous at the three selected frequencies. Such an effect is explained by the role of the wall deformations in contact with the liquid. It is meaningful to compare the estimated longitudinal wavelength predicted by the Langevin's equation with the microchannel width (Figure 5). By working in this frequency range, it is not possible to obtain a fully-developed standing wave within the microchannel. Thus, the acoustic pressure will rely entirely on the vibration of the solid nearby the liquid. If a homogeneous acoustic field is desired, a rigid wall effect or a working frequency in the range of $\mathrm{MHz}$ would be viable solutions. However, at higher frequencies, damping and heat generation, mainly in the solids, become significant and should not be disregarded. In any case, it should be mentioned that high frequencies (in the $\mathrm{MHz}$ scale) could be of no use for preventing the agglomeration of solid catalysts. The current 2D simulation results illustrate the limits of the Langevin's equation accounting for a simpler geometry without border effects, noticeable at the ends of the piezo in Figure 7B. Indeed, the additional resonances (at 41.7 and $59.0 \mathrm{kHz}$ ) are the result of an interactive coupling between the working liquid and the solid (Figure 7A and C). It is worth noting that in these simulations we did 
A Freq. $41.7 \mathrm{kHz}$ :Total displacement (grey, $\mu \mathrm{m}$ ) - Acoustic pressure (colour, $\mathrm{Pa}$ )

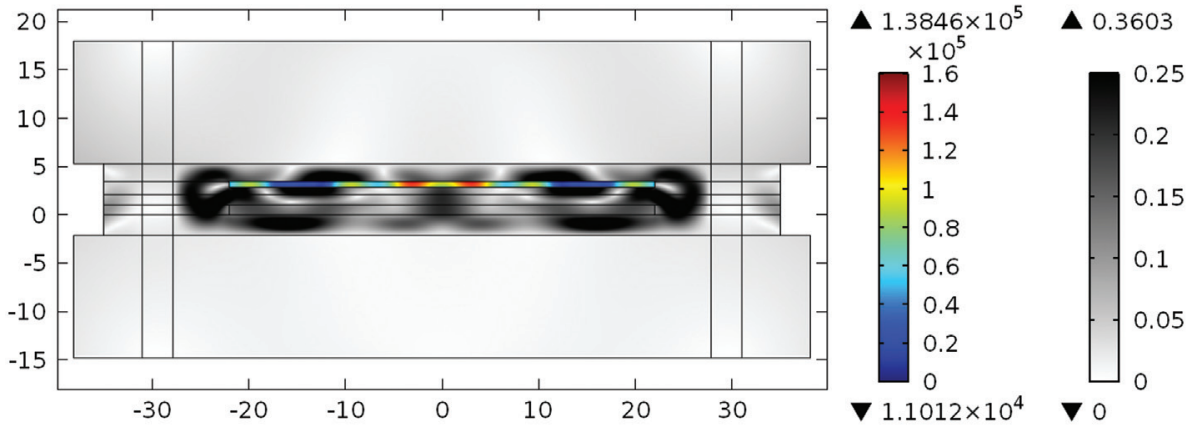

B Freq. $53.5 \mathrm{kHz}$ :Total displacement (grey, $\mu \mathrm{m}$ ) - Acoustic pressure (colour, Pa)

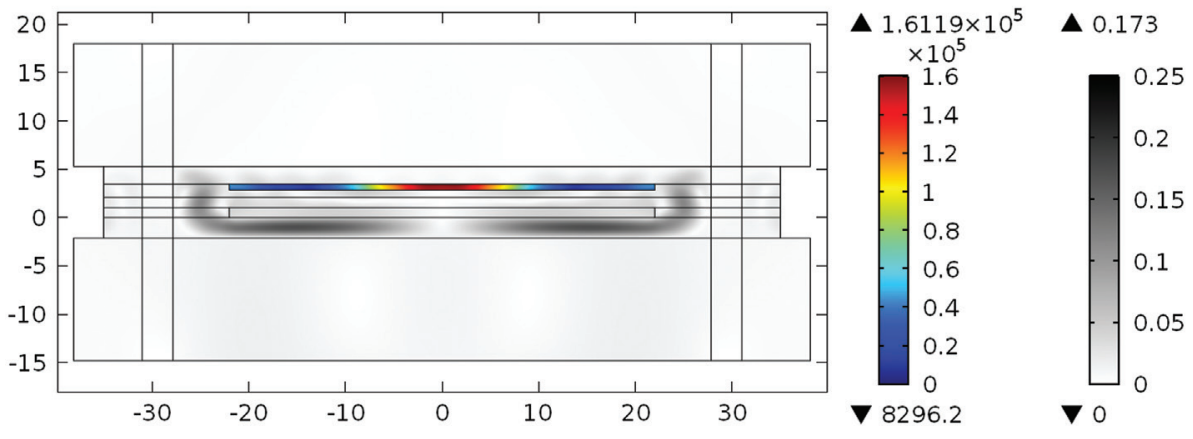

C Freq. $59.0 \mathrm{kHz}$ :Total displacement (grey, $\mu \mathrm{m}$ ) - Acoustic pressure (colour, $\mathrm{Pa}$ )

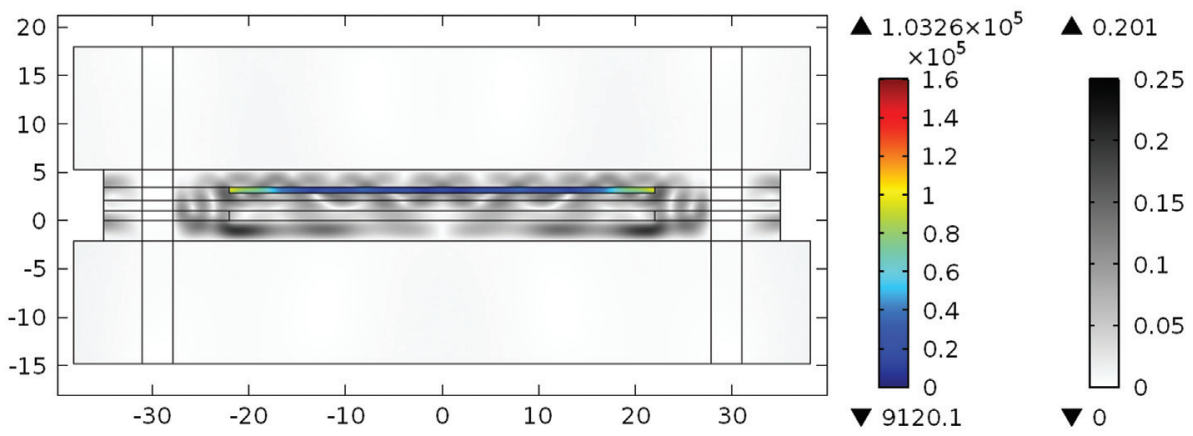

Figure 7 Total displacement and acoustic pressure distribution at different resonant frequencies (A: $41.7 \mathrm{kHz} ; \mathrm{B}: 53.5 \mathrm{kHz}, \mathrm{C}: 59.0 \mathrm{kHz})$ where maximum acoustic pressure is achieved nearby the chosen frequency design $(50 \mathrm{kHz})$.

not introduce acoustic attenuation in the liquid and, thus, the order of magnitude of the acoustic pressure obtained may be too high. Therefore, although this linear-based 2D model shows some agreement with the experimental results in [6], our simulations should be considered on a qualitative or semiquantitative basis rather than on a quantitative one.

We would like to emphasize that despite the limitations faced by using equivalent circuit analogies, they are able to provide crucial information for the efficient design of sono-microreactors. In this sense, the influence of the operating conditions (working frequency) and a proper acoustic sizing (distance between the transducer and the working area) are mandatory steps in the proper design of the devices. Following the proposed strategy (Figure 1), numerical simulations can provide an in-depth understanding of the device physical behavior. Yet, some limitations arise with this approach as well. In fact, not considering non-linear phenomena - or at least a more rigorous treatment of their effects - accounting for cavitation bubbles, structure pre-stress, etc. may cast doubt on our numerical approach. Therefore, such limitations, constituting main challenges in this area of research, should be examined and addressed. As pointed out in recent reviews concerning the simulation of sonochemical systems $[15,26]$, modeling including cavitation can be 
carried out through the so-called continuum and particle approaches. The continuum approach assumes the bubble distribution as a continuous function of time, space, and bubble size. The particle approach considers the bubbles as particles and takes into account their interaction with the acoustic field and among them, as well as mass and viscous drag forces. In any case, as pointed out by Louisnard and González-García [15], simple simulations based on linear acoustics can be profitably used for sonoreactor design. Within this context, the strategy proposed in this work enables to choose the desired working frequency range according to the particular needs of the system before designing and mounting the device. We believe that it constitutes indeed a step forward for the design of efficient sono-microreactors.

\section{Conclusions}

The use of microreactors allows for the development of green production methods because of their higher selectivity and reduced amount of waste. However, one of their main drawbacks is that the device frequently needs to cope with the formation of precipitates within the microchannels. The present work is motivated by the potential advantages that the incorporation of ultrasound irradiation offers for preventing microreactor-related problems and for enhancing their performance. Concretely, an optimization-oriented microreactor sizing is proposed based on the use of a simple acoustic model, leading to approximate results in the design process. Importantly, our calculations help rationalize experimental results found in the literature [6]. The simplicity of the onedimensional model presented here must be emphasized. The Langevin's equation is the simplest analytical model obtained when using the electromechanical equivalent circuit approach. The inherent limitations that this model shows can be partially overcome by performing numerical simulations, which may provide not only a refined sizing but additional information on the acoustic field intensity and distribution. Further work is underway to extend the complexity of the strategy shown here to other types of sono-microreactors, as well as to achieve their verification through two and three-dimensional acoustic simulations (eventually accounting for the presence of bubbles).

We want to highlight that a proper acoustic design of the microreactor is preferable over an empirical optimization of the working frequency in a microreactor where the actuator is included $a$ posteriori. In fact, the physicochemical effects of ultrasound depend on the frequency being employed. Therefore it is important to work at specific frequencies, which demands taking the acoustic design as a crucial part of the sono-microreactor design.

Acknowledgments: This research is funded by the EU project MAPSYN: Microwave, Acoustic and Plasma SYNtheses, under grant agreement No. CP-IP 309376 of the European Union Seventh Framework Program.

\section{References}

[1] Jähnisch K, Hessel V, Löwe H, Baerns M. Angew. Chem. Int. Ed. 2004, 43, 406-446.

[2] Hessel V, Cravotto G, Fitzpatrick P, Patil B, Lang J, Bonrath W. Chem. Eng. Process. 2013, 71, 19-30.

[3] Sedelmeier J, Ley SV, Baxendale IR, Baumann M. Org. Lett. 2010, 12, 3618-3621.

[4] Hartman RL, Naber JR, Zaborenko N, Buchwald SL, Jensen KF. Org. Process Res. Dev. 2010, 14, 1347-1357.

[5] Horie T, Sumino M, Tanaka T, Matsushita Y, Ichimura T, Yoshida Ji. Org. Process Res. Dev. 2010, 14, 405-410.

[6] Kuhn S, Noël T, Gu L, Heider PL, Jensen KF. Lab Chip 2011, 11, 2488.

[7] Hartman RL. Org. Process Res. Dev. 2012, 16, 870-887.

[8] Castro F, Kuhn S, Jensen K, Ferreira A, Rocha F, Vicente A, Teixeira J. Chem. Eng. J. 2013, 215-216, 979-987.

[9] Wu K, Kuhn S. Chim. Oggi 2014, 32, 62-66.

[10] Aljbour S, Yamada H, Tagawa T. Chem. Eng. Process. 2009, 48, 1167-1172.

[11] Fernandez Rivas D, Cintas P, Gardeniers HJGE. Chem. Commun. (Cambridge, U. K.) 2012, 48, 10935-10947.

[12] lida Y, Yasui K, Tuziuti T, Sivakumar M, Endo Y. Chem. Commun. 2004, 20, 2280.

[13] Tandiono, Ohl SW, Ow DSW, Klaseboer E, Wong VVT, Camattari A, Ohl CD. Lab Chip 2010, 10, 1848.

[14] Tandiono T, Siak-Wei Ow D, Driessen L, Sze-Hui Chin C, Klaseboer E, Boon-Hwa Choo A, Ohl SW, Ohl CD. Lab Chip 2012, 12, 780-786.

[15] Louisnard O, González-García J, Feng H, Barbosa-Canovas G, Weiss J, Eds., Ultrasound Technologies for Food and Bioprocessing, Food Engineering Series Springer: New York, 2011.

[16] Mason T, Cobley A, Graves J, Morgan D. Ultrason. Sonochem. 2011, 18, 226-230.

[17] Minchenko H. IEEE Trans. Sonics Ultrason. 1969, 16, 126-131.

[18] Neppiras E. Ultrasonics Int. Conf. Proc. 1973, 295-301.

[19] Shuyu L, Fucheng Z. Ultrason. 1994, 32, 39-42.

[20] Radmanovic M, Mancic D. In Design and Modeling of the Power Ultrasonic Transducers. Faculty of Electronics, Ed., University of Nis, Nis: Serbia 2004.

[21] Shuyu L. J. Acoust. Soc. Am. 2004, 115, 182-186.

[22] Lin S. IEEE Trans. Ultrason. Ferroelectr. Freq. Control 2009, 56, 1990-1998.

[23] Sherrit S, Leary S, Dolgin B, Bar-Cohen Y. Ultrasonics Symposium, 1999, 2, 921-926. 
[24] Al-Budairi H, Lucas M, Harkness P. Sens. Actuators, A 2013 , 198, 99-106.

[25] Mathieson A, Cardoni A, Cerisola N, Lucas M. IEEE Trans. Ultrason. Ferroelectr. Freq. Control 2013, 60, 1126-1133.

[26] Tudela I, Sáez V, Esclapez MD, Díez-García MI, Bonete P, González-García J. Ultrason. Sonochem. 2014, 21, 909-919.

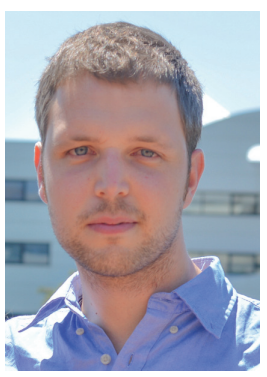

Francisco J. Navarro-Brull, Dipl.-Ing. in Chemical Engineering and MA in Material Science, is currently a PhD candidate at the University of Alicante (Spain). Simultaneously, he researches within the framework of a European Project called MAPSYN, in which he is responsible for the mathematical modeling and computer multiphysics simulation of microreactors. Previously, during his studies, he also worked for a year at Bayer Technology Services (Germany) as a data analyst.

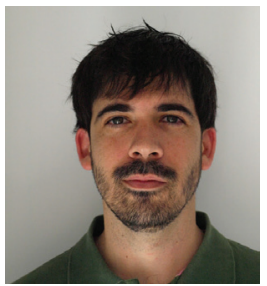

Dipl.-Ing. Pedro Poveda studied Industrial Engineering at Universitat Miguel Hernández d'Elx, Alacant, where he obtained his degree in 2004. In 2011 he finished his BS in Telecommunication Engineering at Universitat d'Alacant, specializing in image and sound. He received an MBA from the EOI Business School and a Master's in Automation and Robotics from the Universitat d'Alacant, he works at the Laboratory of Applied Acoustics at the Deptartment of Physics, Systems Engineering and Signal Theory at the University of Alicante.

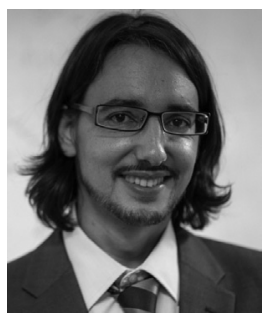

Rubén Ruiz-Femenía is an Associate Professor at the Department of Chemical Engineering at the University of Alicante (Spain). He is member of the research group COnCEPT (Computer Optimization of Chemical Engineering Processes and Technologies). His research interests include modelling and optimization of chemical processes following different approaches from flowsheet structural optimization to detailed modelling using finite element analysis.
[27] Louisnard O, González-García J, Tudela I, Klima J, Sáez V, Vargas-Hernandez Y. Ultrason. Sonochem. 2009, 16, 250-259.

[28] Tudela I, Sáez V, Esclapez MD, Bonete P, Harzali H, Baillon F, González-García J, Louisnard O. Chem. Eng. J. 2011, 171, 81-91.

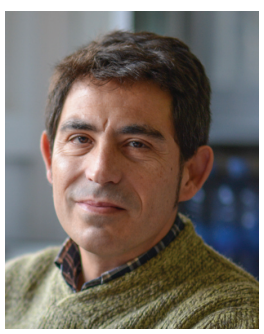

Pedro Bonete obtained his diploma in Chemistry in 1990 and received his PhD from the University of Alicante in 1995 where he worked on the chemistry of stabilized carbanions. After postdoctoral research work on electrochemical synthesis of solvated electron solutions he became an Assistant Professor of Physical Chemistry. His research interests include the coupling of ultrasound and electrochemistry for synthesis and waste water treatment and the electrochemistry of semiconductors.

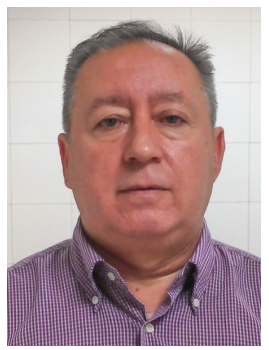

Jaime Ramis Soriano is a Professor at the University of Alicante where he teaches in the fields of acoustic engineering, architectural acoustics and electroacoustics. As a researcher, he has been part of collaboration projects with different companies for the development and improvement of the performance of transducers and loudspeakers.

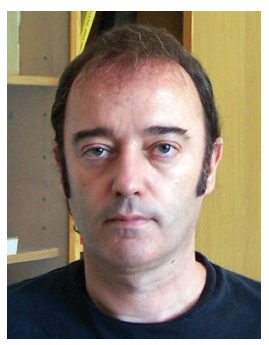

Roberto Gómez is an Associate Professor of Physical Chemistry at the University of Alicante where he leads the Group of Photochemistry and Electrochemistry of Semiconductors. His research has focused on several aspects of fundamental and applied electrochemistry, working with both metals and semiconductors. In recent years he has become interested in the intensification of chemical processes by means of a combination of ultrasound and microreactors. 